\title{
Method to Visualise and Measure Individual Modes in a Few Moded Fibre.
}

\author{
I.P. Giles ${ }^{(1)}$, A. Obeysekara ${ }^{2)}$, F. Poletti(2), D. J. Richardson ${ }^{(2)}$ \\ (1) Phoenix Photonics Limited, UK ian.giles@phoenixphotonics.com \\ (2) Optoelectronics Research Centre, University of Southampton, UK
}

\begin{abstract}
Coupling between the propagating modes and radiation modes of a FMF enables separation and measurement of the properties of the light in each mode independently. A method using prism coupling from a side-polished fibre is described to access and select individual modes.
\end{abstract}

\begin{abstract}
Introduction
Mode Division Multiplexing (MDM) using Few Mode Fibre (FMF) is emerging as a promising approach to increase the capacity of a single fibre. Systems experiments using FMF have shown interesting results with the potential to offer a solution for next generation networks ${ }^{1}$. As with any system the primary building blocks are the components, modules and sub-systems that comprise the whole. Functionality provided by a component may not be identical for all modes and should be characterised for each of the propagating modes. Component development therefore relies on the ability to measure device performance for each of the modes independently. There are methods available to identify the modal content in a fibre, but none provide real time information critical during component fabrication ${ }^{2,3}$. We propose and describe a concept to separate the modes to enable individual modal analysis, providing real time monitoring during component fabrication. The approach investigated is targeted at device performance measurement, but has the flexibility for application in Optical Channel Monitoring (OCM).
\end{abstract}

\section{Concept}

Each mode propagating along the FMF has a different phase velocity and consequently different effective refractive index. When coupled to radiation modes the light will radiate at an angle dependent on the effective index, each mode will therefore radiate at a different angle. One method previously described utilises tilted Bragg gratings written into the FMF to couple light to the radiation modes ${ }^{4}$. The approach taken here is to gain access to the evanescent field of the modes and couple to the radiation modes using a high index prism.

\section{Side-polished fibre}

The evanescent field of the modes can be accessed through a variety of methods by tapering or etching the fibre. The approach selected here offers a very controllable robust method. The cladding material can be removed from one side of the fibre by grinding and polishing over a rotating wheel. The amount of cladding removed and the length of fibre processed can be accurately controlled. Therefore the level of penetration into the evanescent field of each of the modes can be controlled. The finished surface is flat and runs parallel to the core facilitating a controllable interaction length. A prism attached to the sidepolished fibre (figure 1) will couple out the modes at an angle $\left(\theta_{m}\right)$ given by $\sin \left(\theta_{m}\right)=\left(n_{p}{ }^{2}\right.$ $\left.n_{e m}{ }^{2}\right)^{1 / 2}$ where $n_{p}$ is the prism refractive index and $n_{e m}$ is the effective index of the $m^{\text {th }}$ mode coupled out ${ }^{5}$.

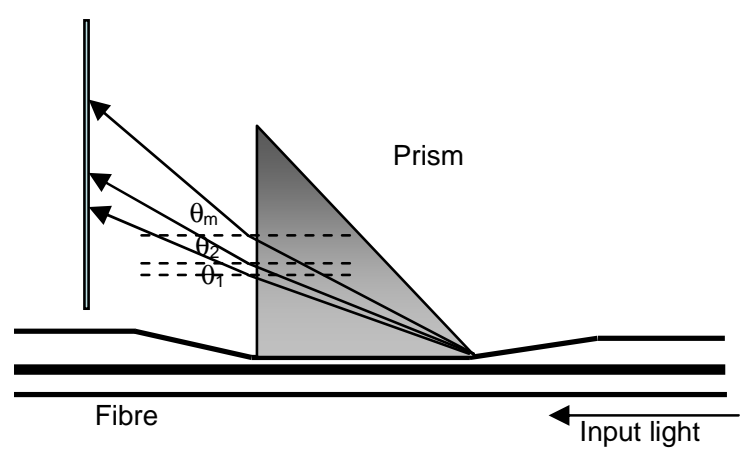

Figure 1 - Schematic of prism coupler on side-polished fibre; the angle of the radiated modes depends on the effective index giving a spatial separation of the modes.

\section{Few mode fibre}

Initial proof of concept work was undertaken on FMF fabricated by OFS Denmark as part of the MODE-GAP project ${ }^{6}$. Initially work was focussed on the dual moded fibre propagating $\mathrm{LP}_{01}$ and $\mathrm{LP}_{11}$ modes to establish the practicality of the concept. The properties of the fibre were well understood and the effective refractive indices were known. Figure 2 shows the calculated radiation angles for the $L P_{01}$ and $L P_{11}$ modes for a BK7 prism attached to a side polished dual mode fibre. Based on the calculated values $\mathrm{LP}_{01}$ couples at $\sim 24^{0}$ at $1550 \mathrm{~nm}$ and the angular difference between the two modes is $0.28^{\circ}$ which is sufficient to spatially separate the modes for detection and analysis. 
BK7 glass prism mode angles OFS DM fibre

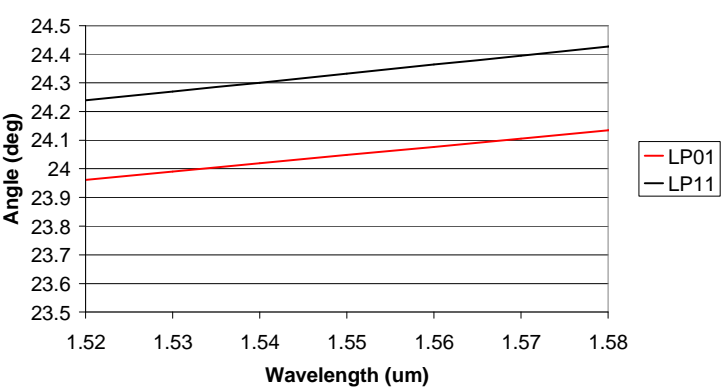

Figure 2 - Graph showing the radiation angles with wavelength for $L P_{01}$ and $L P_{11}$ modes.

\section{Prism mode selector}

The fibre was polished over a rotating wheel to within a few microns of the core. The polished section was $\sim 15 \mathrm{~mm}$ long, the profile tapered to a flat surface parallel to the core providing a good surface to interface with a prism. The fibre was set face up in a v-groove and a $5 \mathrm{~mm}$ prism attached using UV curing high index epoxy. Prism couplers were fabricated on dual mode fibre (DMF) and standard SMF28 single mode fibre. Figure 3 shows the prism coupler and the radiated modes from the SMF28 fibre with $630 \mathrm{~nm}$ light. The crescent shape of the image for each of the modes can be clearly seen. This technique enabled direct visualisation of the output to ensure correct alignment of the fibre and prism.
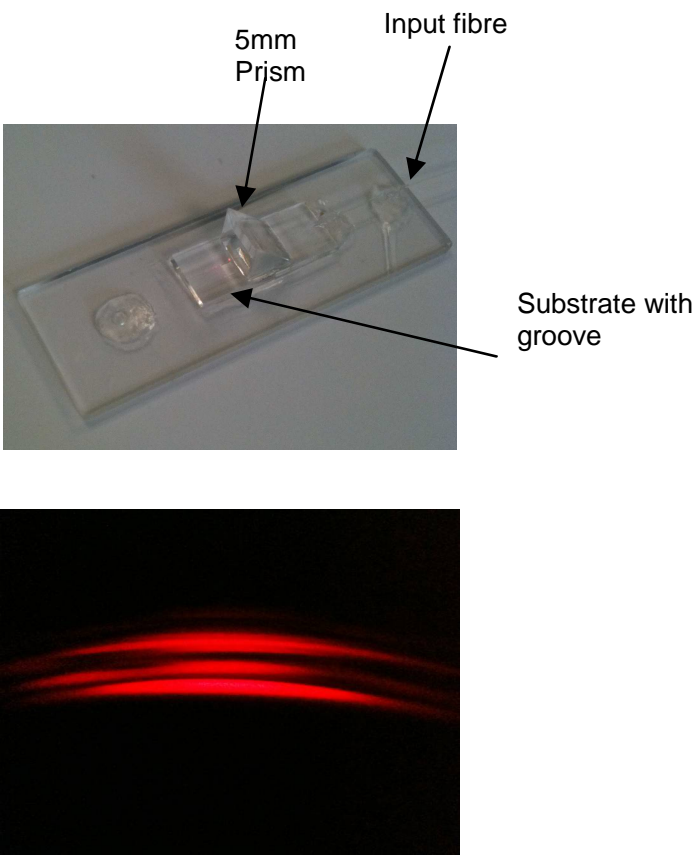

Figure 3 Prism coupler and radiated modes from $630 \mathrm{~nm}$ light into SMF28 fibre with most power in first three modes.

Images of the modes can be visualised and are clearly separate, however to analyse each mode independently the radiated light should be spatially filtered. Figure 4 shows the experimental set-up to evaluate the 2-mode fibre to separate $\mathrm{LP}_{01}$ and $\mathrm{LP}_{11}$ modes. The system consists of a tunable laser source input to a polarization controller in SMF. The SMF is spliced into the dual mode fibre (DMF) to optimise $\mathrm{LP}_{01}$ launched followed by a mode stripper to further filter the $\mathrm{LP}_{01}$ mode. $\mathrm{A}$ mechanical long period grating (LPG) mode converter ${ }^{7}$ was used to adjust between $\mathrm{LP}_{01}$ and $\mathrm{LP}_{11}$ modes. The prism was mounted on an adjustable mount to align to the detection system.

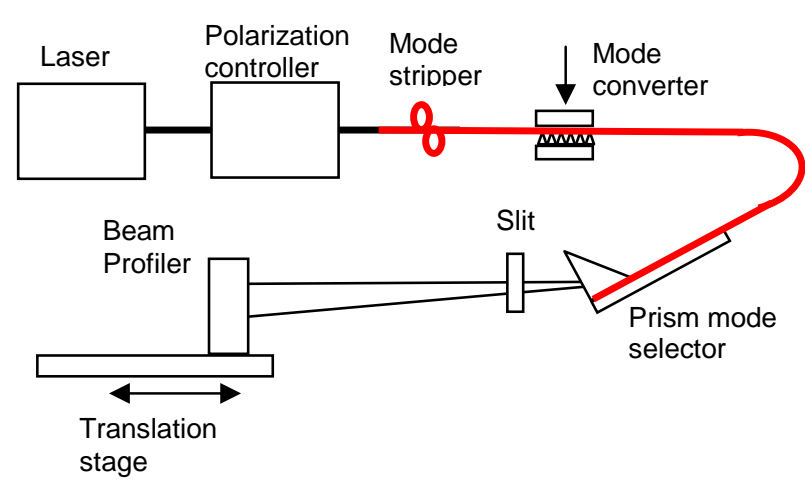

Figure 4 Prism mode selector test set-up showing option of imaging the radiated modes for spatial and power measurement on a beam profiler.

The output could be imaged on a beam profiler that was mounted on a translation stage. The profiler provided spatial positioning of the beam to align the $\mathrm{LP}_{01}$ mode and using the translation stage the divergence angle between $\mathrm{LP}_{01}$ and $\mathrm{LP}_{11}$ was measured to be $0.29^{\circ}$ which was in line with the calculated value for the fibre.

The modal filtering created a pure $\mathrm{LP}_{01}$ in the DMF. The mechanical LPG was designed to apply pressure to the fibre and the $\mathrm{LP}_{11}$ mode conversion could be controlled to $30 \mathrm{~dB}$ at the measurement wavelength. Applying pressure to the mechanical LPG increased the coupling from $L P_{01}$ to $L P_{11}$ until a maximum coupling was achieved. Figure 5 shows images for three conditions; $\mathrm{LP}_{01}, \mathrm{LP}_{01} \& \mathrm{LP}_{11}, \mathrm{LP}_{11}$.
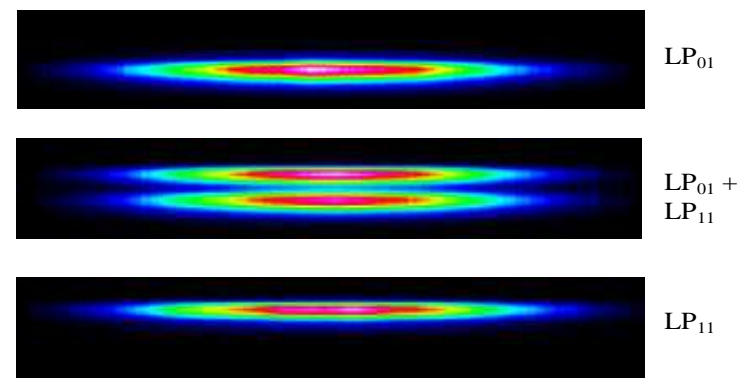

Figure 5 Photographs of prism selector images for $L P_{01}$ and $L P_{11}$ (Note: non-crescent shape is due to beam profiler processing) 
Using a simple variable slit spatial filter the relative isolation of the two modes was measured. Masking the $\mathrm{LP}_{01}$ mode and varying the LPG pressure an isolation of $>17 \mathrm{~dB}$ was achievable (figure 6). The isolation when masking $\mathrm{LP}_{11}$ was lower due to the crescent shape of the image. Improved isolation will be achievable with spatial filtering specific to the image shape.

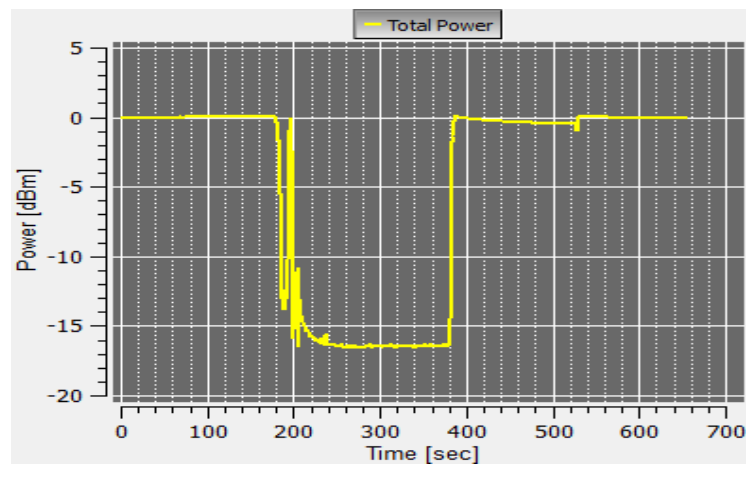

Figure $6 L P_{01}$ output blocked: $L P G$ tuned between maximum $\left(L P_{11}\right)$ and minimum $\left(L P_{01}\right)$ detected power.

Varying the input state of polarization (SOP) the LPG affects the spatial mode generated varying from $L P_{11 a}$ to $L P_{11 b}{ }^{7}$. The SOP was varied into the prism mode selector to observe changes to the image. When a pure $L_{01}$ mode was transmitted variation of the SOP did not alter the power detected or the shape of the image. Changing polarization state with a pure $\mathrm{LP}_{11}$ mode showed marked change in image and coupled power. There were two clearly defined states shown in figure 7 and various changes between the two.
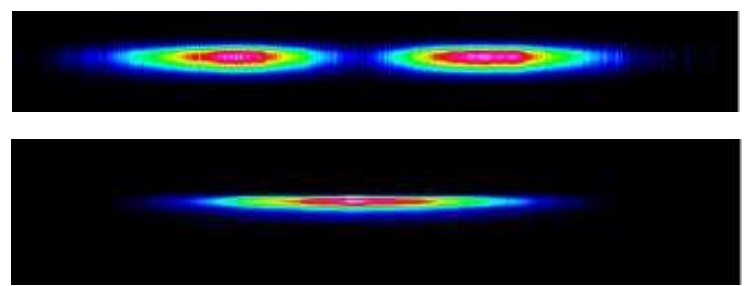

Figure $7 L P_{11}$ modal images for two different input polarization states representing different $L P_{11}$ mode orientation

\section{Discussion and conclusions}

Side polishing a fibre to access the evanescent field of the propagating modes and coupling to the radiation modes through a high index prism enables imaging of the divergent modes. The principle has been investigated using a dual mode fibre separating the $\mathrm{LP}_{01}$ and $\mathrm{LP}_{11}$ modes and measuring the relative powers in each of the modes. Simple spatial filtering techniques allow separation of the modes and more complex methods will improve isolation. By altering the input SOP to a variable mechanical LPG mode converter the orientation of the LP 11 mode can be modified. The radiated images are changed due to the variation in orientation showing two distinct patterns related to the orientation of the mode.

Using the side-polishing technique to remove the cladding gives full control over the amount of cladding removed and the length of fibre over which it is removed. The mode selector can be designed to couple out a specified fraction of power from the fibre offering a range of additional potential applications.

Isolating the radiated modes provides a method to monitor component output during manufacture and measure modal parameters following fabrication. Additional applications such as OCM are also feasible.

\section{Acknowledgments}

This work is partly supported by EU program FP7-ICT-258033 MODE-GAP. Asiri Obeysekara is supported by a UK EPSRC CASE studentship in conjunction with Phoenix Photonics. Few moded fiber designed and provided by OFS Denmark as part of the MODE-GAP project.

\section{References}

[1] A.Li et al., "Reception of Mode and Polarization multiplexed 107-Gb.s CO-OFDM Signal over two moded fiber." OFC/NFOEC post deadline PDPB8 (March 2011)

[2] J.W. Nicholson, et al., "Measuring the Modal Content of Large-Mode-Area Fibers." Quantum, 15(1), 61-70 (2009)

[3] O.Shapira, et al., "Complete Modal Decomposition for Optical Waveguides." Physical Review Letters, 94(14), 1-4. (2005)

[4] L.Yan et al., "Side-tap modal channel monitor for mode division multiplexed (MDM) systems" OFC/NFOEC OM3C.2 (March 2012)

[5] W.V.Sorin et al., "Phase-velocity measurements using prism output coupling for single- and few-mode optical fibers." Optics Letters, Vol. 11, No. 2 (1986)

[6] EU FP7-ICT-25033 MODE-GAP www.modegap.eu

[7] I.P.Giles et al. "All Fiber Components for Multimode SDM Systems" IEEE Summer Topical conference on SDM (July 2012). 